\title{
Etiological diagnosis of pneumonia: A goal worth pursuing?
}

$\mathrm{P}$ NEUMONIA IS A COMMON DISEASE, AND IDENTIFICATION OF responsible pathogens should lead to a reduction of still unacceptably high mortality and morbidity rates. However, the extent of diagnostic efforts should be tailored to the severity of illness. In view of the high incidence of community acquired pneumonia (CAP) and its attendant morbidity and mortality ( 1 to $5 \%$ in out-patients and $25 \%$ in in-patients), the importance of a correct etiological diagnosis and appropriate antimicrobial therapy becomes evident (1). Rapid investigations, with high positive and negative predictive values, are necessary (2). Despite extensive, costly investigation in carefully performed prospective studies, an etiological diagnosis is made in only $50 \%$ of cases (3). The necessity of exhaustive attempts to diagnose CAP has been questioned (1).

Clinical acumen has long been touted as critical to making a bacteriological diagnosis of pneumonia, especially in major texts $(4,5)$. Fang et al $(6)$, analyzing 22 common symptoms, signs and laboratory findings in pneumonia, did not find any features that helped distinguish among the five most common pathogens (Streptococcus pneumoniae, Haemophilus influenzae, Legionella species, Chlamydia pneumoniae or Gramnegative rods). The so-called 'atypical' presentation of pneumonia (nonproductive cough, viral prodrome, lack of consolidation) was not unique to the 'atypical' pathogens. The overlap of clinical presentation of these pathogens precluded precise recommendations for specific antimicrobial therapy. The manifestations of pneumonia are more a reflection of the host response than of the inciting microorganism.

Woodhead et al (2) found that the results of routine tests (such as sputum Gram stain and culture, blood cultures and serology) changed management in only $8 \%$ of patients. Since $30 \%$ of patients cannot produce sputum, another $30 \%$ have previously been treated with antibiotics and $25 \%$ have organisms that are not easily cultured, the use of sputum Gram stain and culture as diagnostic tools is limited (7). Under ideal circumstances, a sensitivity of $62 \%$ and a specificity of $85 \%$ have been reported; however, under less than ideal circumstances (the 'real world'), these values can be as low as $50 \%$ and $12 \%$, respectively (8). Sputum cultures are potentially misleading in that organisms isolated may be pathogens or colonizers (3). In proven pneumococcal pneumonia, sputum cultures have a $45 \%$ false negative result (9). However, sputum Gram stain and culture can diagnose organisms not normally present in the respiratory tract, such as Mycobacterium tuberculosis, Pneumocystis carinii and histoplasma, and can possibly identify resistant organisms (1).

Blood cultures are positive in only 7 to $25 \%$ of pneumonias, most commonly in those of pneumococcal origin $(7,10,11)$. Routine blood tests (white blood cell count, electrolytes, liver and renal function tests) provide important prognostic information but are not helpful in a diagnostic sense (1).

The utility of chest roentgenograms has also been disappointing (12). No distinctive roentgenographic pattern can consistently distinguish among pneumococcal, legionella, chlamydial and mycoplasmal pneumonias. Rather than identifying a causative organism, chest roentgenograms should be used to assess the severity of disease and complications such as abscesses, obstruction, effusions, etc (1).

Serological antibody tests were initially viewed as a panacea to the clinician's diagnostic woes. On the contrary, they are of limited availability, costly, have low yield (25\%) and, because of processing delay, cannot affect patient treatment in the critical early period (3). Their use should be limited to special situations, such as epidemiological surveys of pneumonia.

For pneumococcal infection, microbial antigens, such as pneumococcal $\mathrm{C}$ polysaccharide and capsular polysaccharide, can be found in sputum, serum and urine (10). However, these tests are expensive, have varying sensitivities and specificities according to the source examined and to the diagnostic modality used (ELISA or countercurrent immunoelectrophoresis, for example), and results are not immediately available. Furthermore, serum and urine antigen positivity persists up to six months after an infection, further lowering its specificity. Hence, these investigations have not been widely accepted.

Invasive procedures, such as transtracheal aspiration, protected brush, protected bronchoalveolar lavage (BAL), needle lung aspiration and open biopsy are not indicated in CAP unless the patient is severely ill, because these procedures rarely aid rapid diagnosis and expose the patient to unnecessary risk (1). 
Despite much effort, few investigations or clinical pearls have been of definitive value in making an etiological diagnosis of CAP and in tailoring antibiotic therapy appropriately.

Patients should be treated empirically, therefore, according to the most likely cause of infection, depending on their age, co-morbid diseases, severity of illness and need for hospitalization $(1,3)$. Extensive work-up should be limited to sick nonresponders. Further progress in diagnostic testing is needed, and perhaps new technologies, such as the polymerase chain reaction, may help fill this void. While accepting the role of empirical therapy, one should not espouse a complacent attitude towards it. Recognition of the evolving spectrum of community pathogens should lead to continuing surveillance epidemiological studies. This infers that practice guidelines should be revisited frequently to allow physicians to keep abreast of the changes in epidemiological patterns.

Immunocompromised patients form a subgroup in whom specific rather than empirical therapy is preferred. There is a $40 \%$ incidence of opportunistic infections in these hosts, leading to a $50 \%$ mortality rate (13). Invasive investigations are thus important for definitive diagnosis. The sensitivity and specificity of these procedures vary according to the type of immunosuppression and the prophylactic or empirical therapy already received (14). Localized processes can be treated empirically initially, whereas diffuse disease generally necessitates a diagnostic procedure $(13,14)$. Williams et al (13) found fibreoptic bronchoscopy (FOB) with brushings, lavage and transbronchial biopsy to be diagnostic in $58 \%$ of patients, a value similar to that obtained by Crocket et al in this issue of the Journal (pages 286-290). FOB was $90 \%$ sensitive in diagnosing an infectious process; although transbronchial biopsy does not raise this sensitivity more than brushings alone, it is necessary to rule out noninfectious processes (13). The individual yields of brush, biopsy and lavage are about 30 to $55 \%$; it is in their combined use that the diagnostic yield of bronchoscopy can rise to 80 to $95 \%$ (14). In Williams and colleagues' (13) study, the high negative predictive value of FOB implied that a subsequent open lung biopsy had a low probability of identifying an infectious disease.

Non-intensive care associated nosocomial pneumonia occurs in $1 \%$ of hospitalized patients and is the third most common hospital acquired infection, with a mortality ranging from 16 to $37 \%$ (15). As in CAP, the etiology of nosocomial pneumonia cannot be inferred from clinical or radiological grounds, and empirical therapy should be initiated, with invasive workup being done in nonresponders (15).

Ventilator acquired pneumonia (VAP) is defined as that developing more than $72 \mathrm{~h}$ after intubation (16). It occurs in 10 to $20 \%$ of patients with respiratory failure and in up to $70 \%$ of adult respiratory distress syndrome (ARDS) patients (16). Its mortality of 50 to $80 \%$ (17) further underlines the significance of this disease in critically ill patients and the importance of prompt, effective antibiotic therapy. The consequences of not treating VAP are evident; those of overtreatment, less so. However, excessive use of antibiotics, besides incurring unnecessary costs and exposing the patient to potential side effects and toxicities, leads to colonization and growth of potentially resistant organisms; subsequent superinfection results in higher mortality than that seen in patients not previously on antibiotics $(16,17)$.

There are many difficulties in the clinical diagnosis of VAP. Recognition of pneumonia is usually based on the presence of fever, elevated white blood cell count, purulent secretions and chest roentgenographic abnormalities, criteria that, in the critically ill, can be misleading $(16,17)$. In the intensive care unit setting, many disorders, often not infective in nature, can lead to fever and an elevated white blood cell count; sputum can result from leakage of oropharyngeal secretions around the endotracheal tube; chest roentgenographic changes can be explained by pulmonary edema, atelectasis, pulmonary embolism or ARDS; even positive sputum cultures may simply be from colonization of the oropharynx or from tracheobronchitis $(16,17)$. Clearly, many patients in whom there is a strong suspicion of pneumonia have another disorder. In a study by Fagon et al (18), the use of 16 clinical variables for the diagnosis of pneumonia led to a 30 to $40 \%$ diagnostic error and overtreatment in $16 \%$ of patients. More definitive means of diagnosing pneumonia are evidently needed.

The answer does not lie with noninvasive tests, such as sputum analysis or tracheal aspiration. No correlation has been found between cultures from sputum and from uncontaminated locations (blood, pleural fluid) (16). Tracheal aspirates have a false positive rate of $21 \%$, due to colonization (19). However, in a study by Salata et al (20), the presence of elastin fibres and a high bacterial grading on Gram stain in tracheal secretions had a $100 \%$ positive predictive value and a sensitivity ranging from 52 to $67 \%$, depending on the diagnostic criteria used for pneumonia. This test holds promise, but needs to be validated in a larger clinical study.

Chest roentgenograms are of limited use in the diagnosis of VAP: they are often portable, lack a lateral projection and are improperly positioned (21). Their diagnostic efficiency is less than $70 \%$ and the only useful sign is that of air bronchograms, which predict $64 \%$ of pneumonias (21).

The role of invasive testing for diagnosis of pneumonia is more clear in patients with VAP. The technique of Wimberley et al (22) of protected specimen brush has a sensitivity of 65 to $96 \%$ and a positive predictive value of $75 \%$, significant cultures being those with more than $10^{3}$ colony forming units $(\mathrm{CFU}) / \mathrm{mL}(16,18)$. The negative predictive value approaches $100 \%$; however, the false negative rate is about 5 to $10 \%(23)$. Drawbacks to its use include the small sample size obtained, which may miss an adjacent area of involved lung; the precise methodology (no use of suction or lidocaine) needed to maximize return; the false positive rate of 9 to $25 \%$; and the one- to two-day delay in obtaining final results (24-26).

Even quantitative cultures of BAL fluid, considered significant with a growth of more than $10^{5} \mathrm{CFU} / \mathrm{mL}$, are frequently contaminated from the upper airway (27). In an attempt to minimize this, specimens are excluded from analysis if they contain more than $1 \%$ squamous epithelial cells; this purportedly raises the sensitivity to $88 \%$ and specificity close to $100 \%$ (16). Gram stain can also be done on BAL fluid to quan- 
tify intracellular organisms and thus guide preliminary therapy (27).

The optimal diagnostic strategy in patients with suspected VAP is unknown. There is discordance among techniques, including such crucial variables as the definition of a significant quantitative culture, and there are inconsistency and disagreement regarding the diagnostic gold standard for pneumonia, thus rendering comparison between studies difficult (23). The answer lies in a well designed prospective, randomized study comparing empirical therapy for VAP, with intervention occurring if there is a deterioration or lack of improvement of the clinical course with a rapid invasive investigation strategy, in which treatment is reserved for those with a proven bacteriological etiology. This should incorporate both short term and long term outcomes and resource use, a factor that is of growing importance in today's medical practice. Until this study is performed, debate will continue to rage over the value of early intervention with invasive diagnostic tests.

\section{REFERENCES}

1. Niederman MS, Bas JB, Campbell GD, et al. Guidelines for the initial management of adults with community-acquired pneumonia: diagnosis, assessment of severity, and initial antimicrobial therapy. Am Rev Respir Dis 1993;148:1418-26.

2. Woodhead MA, Arrowsmith J, Chamberlain-Webber R, et al. The value of routine microbial investigation in community-acquired pneumonia. Respir Med 1991;85:313-7.

3. Niederman MS. Empirical therapy of community-acquired pneumonia. Semin Respir Infect 1994;9:192-8.

4. Harrison TR. Principles of Internal Medicine, 12th edn, vol 2. New York: McGraw-Hill, Inc, 1991:1065.

5. Mandell GL, Douglas RG, Benett JE. Principles and Practice of Infectious Diseases, 3rd edn. New York: Churchill Livingstone, 1990:540-2.

6. Fang G-D, Fine M, Orloff J, et al. New and emerging etiologies for community-acquired pneumonia with implications for therapy: a prospective multicenter study of 359 cases. Medicine 1990;69:307-16.

7. Marrie TJ. Community-acquired pneumonia. Clin Infect Dis 1994;18:502-15.

8. Rein MF, Gwaltney JM, O'Brien WM, et al. Accuracy of Gram's stain in identifying pneumococci in sputum. JAMA 1978;239:2671-3.

9. Barrett-Connor $E$. The nonvalue of sputum culture in the diagnosis of pneumococcal pneumonia. Am Rev Respir Dis 1971;103:845-8.

10. Venkatesan P, Macfarlane JT. [Editorial]. Thorax 1992;47:329-31. (Edit)

11. Chalasani NP, Valdecanas MAL, Gopal A, et al. Clinical utility of blood cultures in adult patents with community-acquired pneumonia without defined underlying risks. Chest 1995;108:932-6.

12. Macfarlane JT, Miller AC, Roderick Smith WH, et al. Comparative radiographic features of community acquired Legionnaire's disease, pneumococcal pneumonia, mycoplasma pneumonia, and psittacosis. Thorax 1984;39:28-33.

13. Williams D, Yungbluth M, Adams G, et al. The role of fiberoptic bronchoscopy in the evaluation of immunocompromised hosts with diffuse pulmonary infiltrates. Am Rev Respir Dis 1985;131:880-5.

14. Shelhamer $\mathrm{JH}$. Respiratory disease in the immunosuppressed patient. Ann Intern Med 1992;117:415-31.

15. Niederman MS, Sarosi GA, Glassroth J. Respiratory Infections: A Scientific Basis for Management. Philadelphia: WB Saunders Company, 1994:139-46.

16. Meduri GU. Ventilator-associated pneumonia in patients with respiratory failure: a diagnostic approach. Chest 1990;97:1208-19.

17. Fagon J-Y, Chastre J, Domart $\mathrm{Y}$, et al. Nosocomial pneumonia in patients receiving continuous mechanical ventilation: prospective analysis of 52 episodes with use of a protected specimen brush and quantitative culture techniques. Am Rev Respir Dis 1989;139:877-84.

18. Fagon J-Y, Chastre J, Hance AJ, et al. Evaluation of clinical judgment in the identification and treatment of nosocomial pneumonia in ventilated patients. Chest 1993;103:547-53.

19. Bartlett JG. Diagnostic accuracy of transtracheal aspiration bacteriologic studies. Am Rev Respir Dis 1977;115:777-82.

20. Salata RA, Lederman MM, Shlaes DM, et al. Diagnosis of nosocomial pneumonia in intubated, intensive care unit patients. Am Rev Respir Dis 1987;135:426-32.

21. Wunderink RG, Woldenberg LS, Zeiss J, et al. The radiologic diagnosis of autopsy-proven ventilator-associated pneumonia. Chest 1992;101:458-63.

22. Wimberley N, Faling LJ, Bartlett JG. A fiberoptic bronchoscopy technique to obtain uncontaminated lower airway secretions for bacterial culture. Am Rev Respir Dis 1979;119:337-42.

23. Meduri GU. Diagnosis and differential diagnosis of ventilator-associated pneumonia. Clin Chest Med 1995;16:61-93.

24. Fagon J-Y, Chastre J, Hance AJ, et al. Detection of nosocomial lung infection in ventilated patients: use of a protected brush and quantitative culture techniques in 147 patients. Am Rev Respir Dis 1988;138:110-6.

25. Meduri GU, Beals DH, Maijub AG, et al. Protected bronchoalveolar lavage: a new bronchoscopic technique to retrieve uncontaminated distal airway secretions. Am Rev Respir Dis 1991;143:855-64.

26. Chastre J, Fagon J-Y, Domart Y, et al. Diagnosis of nosocomial pneumonia in intensive care unit patients. Eur J Clin Microbiol Infect Dis 1989;8:35-9.

27. Chastre J, Fagon J-Y, Soler P, et al. Diagnosis of nosocomial bacterial pneumonia in intubated patients undergoing ventilation: comparison of the usefulness of bronchoalveolar lavage and the protected specimen brush. Am J Med 1988;85:499-506.

\author{
Maria Antoniou MD \\ Ronald F Grossman MD FRCPC FACP \\ Division of Respiratory Medicine \\ Mount Sinai Hospital \\ Toronto, Ontario
}




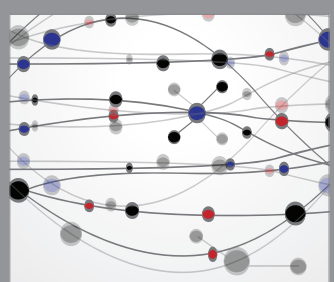

The Scientific World Journal
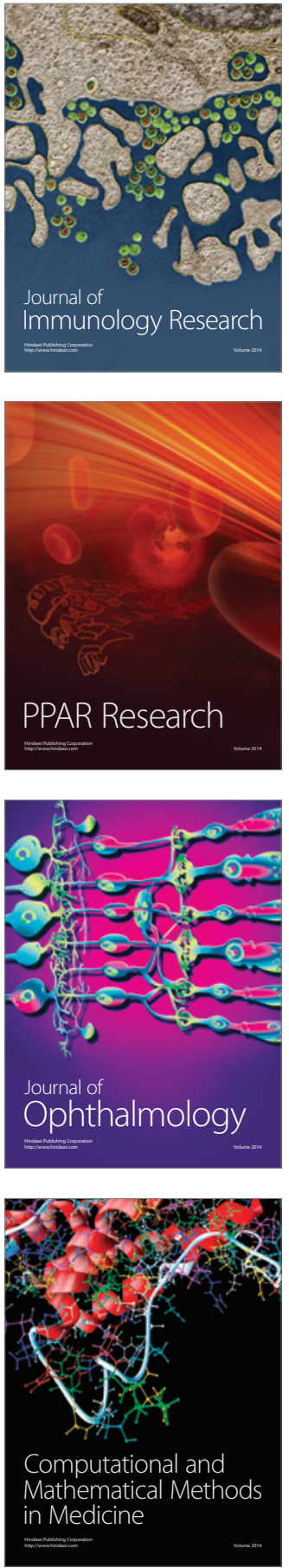

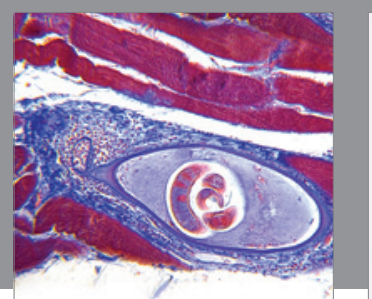

Gastroenterology Research and Practice

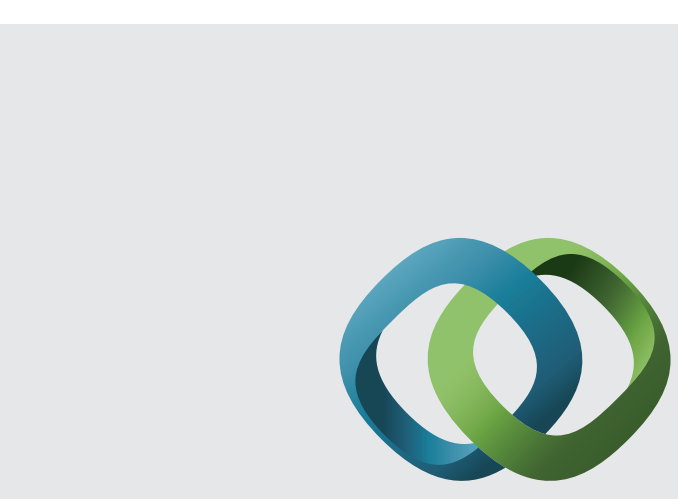

\section{Hindawi}

Submit your manuscripts at

http://www.hindawi.com
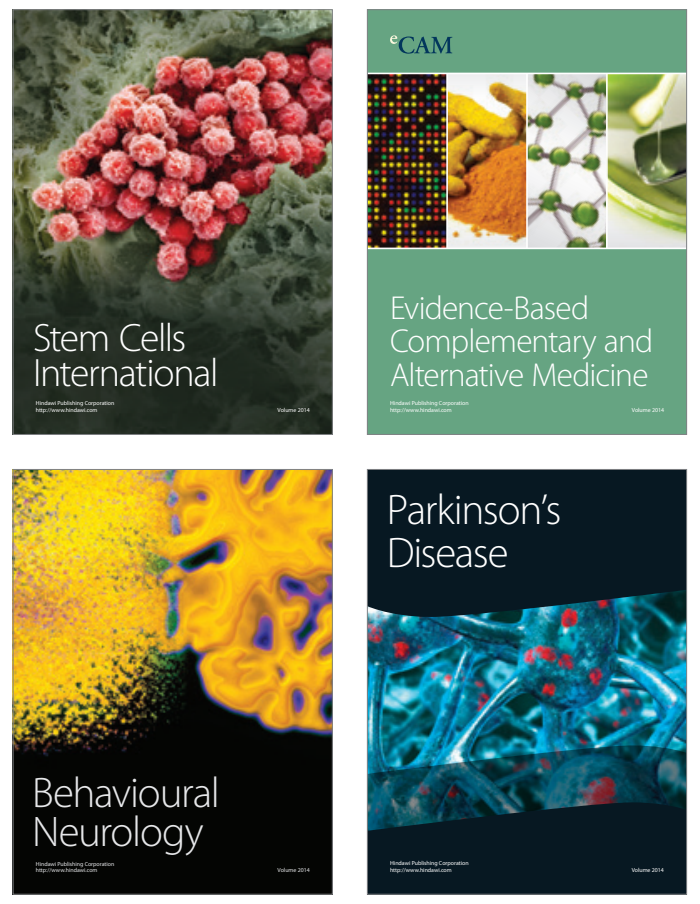
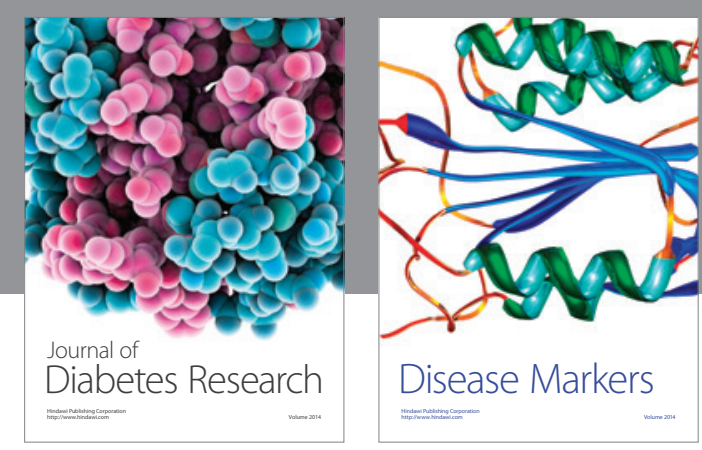

Disease Markers
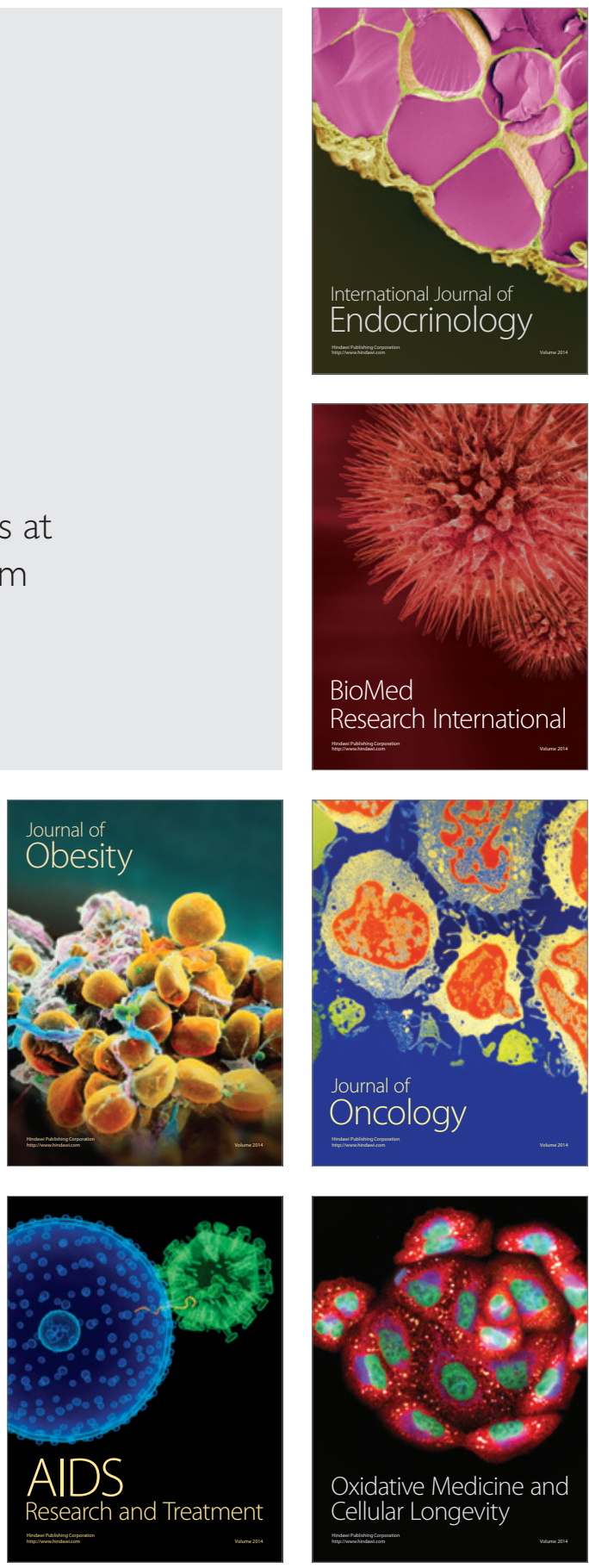